\title{
Does inverse planning improve plan quality in interstitial high-dose-rate breast brachytherapy?
}

\author{
Prof. Tibor Major, PhD, DSc',2, Georgina Fröhlich, PhD'³, Norbert Mészáros, MD',2, Viktor Smanykó, MD!, \\ Prof. Csaba Polgár, MD, PhD, DSl', \\ 'Radiotherapy Centre, National Institute of Oncology, Budapest, Hungary, 2Department of Oncology, Semmelweis University, Budapest, \\ Hungary. ${ }^{3}$ Eötvös Loránd University, Faculty of Science, Budapest, Hungary
}

\begin{abstract}
Purpose: To investigate the effect of input parameters for an inverse optimization algorithm, and dosimetrically evaluate and compare clinical treatment plans made by inverse and forward planning in high-dose-rate interstitial breast implants.

Material and methods: By using a representative breast implant, input parameters responsible for target coverage and dose homogeneity were changed step-by-step, and their optimal values were determined. Then, effects of parameters on dosimetry of normal tissue and organs at risk were investigated. The role of dwell time modulation restriction was also studied. With optimal input parameters, treatment plans of forty-two patients were re-calculated using an inverse optimization algorithm (HIPO). Then, a pair-wise comparison between forward and inverse plans was performed using dose-volume parameters.

Results: To find a compromise between target coverage and dose homogeneity, we recommend using weight factors in the range of 70-90 for minimum dose, and in the range of 10-30 for maximum dose. Maximum dose value of $120 \%$ with a weight factor of 5 is recommended for normal tissue. Dose constraints for organs at risk did not play an important role, and the dwell time gradient restriction had only minor effect on target dosimetry. In clinical treatment plans, at identical target coverage, the inverse planning significantly increased the dose conformality (COIN, 0.75 vs. $0.69, p<0.0001$ ) and improved the homogeneity (DNR, 0.35 vs. $0.39, p=0.0027$ ), as compared to forward planning. All dosimetric parameters for non-target breast, ipsilateral lung, ribs, and heart were significantly better with inverse planning. The most exposed small volumes for skin were less in HIPO plans, but without statistical significance. Volume irradiated by $5 \%$ was $173.5 \mathrm{~cm}^{3}$ in forward and $167.7 \mathrm{~cm}^{3}$ in inverse plans $(p=0.0247)$.

Conclusions: By using appropriate input parameters, inverse planning can provide dosimetrically superior dose distributions over forward planning in interstitial breast implants.

Key words: inverse planning, high-dose-rate, interstitial breast implants.

\section{Purpose}

Inverse planning algorithms have been routinely used in external beam therapy for intensity and volume modulated radiotherapy for several years, but in brachytherapy (BT), it cannot be considered as a standard planning method yet. In BT, the classical dosimetry systems played an important role in standardizing the treatments and were developed for low-dose-rate (LDR) sources. However, the introduction of high-dose-rate (HDR) stepping source technique opened new possibilities in clinical dosimetry. Furthermore, the introduction of cross-sectional imaging and 3D target volume definition in BT required new planning approaches. Forward optimization is now regularly used for BT planning, and the most accepted methods are the geometrical (GO) and graphical optimi- zations (GRO), which can provide clinically acceptable dose distributions for breast implants [1,2,3,4]. During $\mathrm{GO}$, there is no need for defining dose points, because dwell positions themselves serve as reference dose points for optimization. After GO, the dwell time at any dwell position is inversely proportional to the dose delivered by other dwell positions, resulting in homogeneous dose distribution. If the catheters geometrically cover the target volume properly, the planning target volume (PTV) is encompassed by the reference isodose surface. In case of possible geometrical miss of the catheters, an underdosed region can develop in the PTV, which can be decreased using the GRO. With the drag-and-drop method, an isodose line can be shifted into a desired position on the CT image with the cursor on the screen. However, it must
Address for correspondence: Prof. Tibor Major, PhD, DSc, National Institute of Oncology, Radiotherapy Centre, 7-9 Rath Gy. u. 1122 Budapest, Hungary, phone: +36 1224 8600/3277, fax: +361224 8620, 凶e-mail: major@oncol.hu
Received: 21.01 .2020

Accepted: 12.03 .2020

Published: 30.04 .2020 
be remembered that with improving target coverage, the dose homogeneity deteriorates. In BT, the coverage is generally characterized by the $\mathrm{V}_{100}$ (percentage of PTV receiving at least $100 \%$ of the prescribed dose [PD]), while the dose homogeneity is considered as the DNR (dose non-uniformity ratio). The latter is the ratio of volumes irradiated by 1.5 times the PD and the PD. To make an optimal plan, many steps must be occasionally repeated during the planning process, with continuous evaluation of the dose-volume histogram (DVH) parameters. Since the target coverage and dose homogeneity always change in the opposite direction, there is a big challenge for the planner to meet both conditions with a compromise. This requires repetitive dose calculations, which can be time-consuming. On the contrary, during inverse optimization, all dosimetric requirements (dose coverage, dose homogeneity, organs at risk [OARs] protection) are simultaneously and automatically considered. Inverse optimization always requires cross-sectional imaging, since it is anatomy-based, and the 3D volume definitions are its preconditions. The two most frequently used algorithms in BT are the HIPO (hybrid inverse planning optimization) [5] and IPSA (inverse planning simulated annealing) [6].

In BT, apart from prostate cancer, the integration of inverse planning into clinical routine has not been accomplished so far. In the literature, there are reports on the use of inverse planning for cervix cancer $[7,8,9,10,11,12,13]$, vaginal irradiation [14,15], and head and neck brachytherapy $[16,17]$. For breast cancer, the available information is scarce. Among partial breast irradiation techniques, interstitial brachytherapy has the longest patients' follow-up and the highest level of evidence as an alternative treatment option to whole breast irradiation $[18,19,20]$. Based on our experience with forward planning in breast BT, in this work, we performed a detailed investigation about the feasibility of inverse planning in interstitial HDR breast implants and compared its efficacy with conventional forward planning regarding target dosimetry and protection of OARs.

\section{Material and methods}

First, a representative case was selected to investigate the effect of optimization parameters on target dosimetry during inverse optimization. The Oncentra Brachy v.4.5.3 planning system (Elekta Brachytherapy, Veenendaal, The Netherlands) was used for both forward and inverse optimizations. The HIPO implemented in Oncentra Brachy is based on dose-volume parameters defined as minimum (MinValue) and maximum (MaxValue) dose limits for targets $[5,21]$, and for OARs, only maximum dose limits are considered. Based on these dose limits, HIPO calculates a corresponding objective function, and with an importance factor or penalty (MinWeight, MaxWeight) defined by the user, HIPO calculates the total objective function, which is then considered for the optimization. The total objective function is subsequently calculated as the weighted sum (aggregation) of all individual objective functions. The results of optimization are the source dwell times.

Using HIPO, the effects of different weight factors and dwell time modulation on target dosimetry were in- vestigated in detail and subsequently, their optimal values were defined. As a first step, the minimum weight (MinWeight) was changed between 0 and 100 in steps of 10 , and its effect on $V_{100}$ and DNR was calculated at various dose homogeneity. Then, the maximum weight (MaxWeight) was changed between 0 and 100 in steps of 10 , and $\mathrm{V}_{100}$ and DNR were calculated at different target coverage. Afterwards, the optimization on normal tissue and OARs was studied. For normal tissue, the role of maximum dose value and its weight were examined. The objectives used for an OAR situated close to and far from the PTV (skin and rib, respectively) were also changed, and subsequent target dosimetry was assessed. The target volume was adjacent to skin and a few $\mathrm{cm}$ away from the rib.

HIPO includes an option for modulating dwell times in neighboring dwell positions, which is called dwell time gradient restriction (DTGR). The DTGR parameter takes value between 0.0 and 1.0. At value of 0.0 , there is no restriction, and large fluctuations between neighboring dwell times can develop. When maximal value is applied (DTGR $=1.0)$, the resulting distribution of dwell times in catheters will be smooth, similar to GO. The effect of modulation of neighboring dwell times on target coverage and dose homogeneity in HIPO was also investigated. The parameter of DTGR was changed between 0.0 to 1.0 in steps of 0.1 , and $V_{100}$ and DNR were calculated.

In the second part of the study, treatment plans of forty-two patients with early stage breast cancer who were treated with accelerated partial breast irradiation using multicatheter interstitial brachytherapy were selected. The patient selection and target volume definition were based on the GEC-ESTRO recommendations [22,23]. Using the optimal input parameters determined in the first part of our study, competing treatment plans were made with HIPO, and they were compared with the original forward plans.

\section{Implantation and forward planning}

Our implantation technique was based on two CT imaging. The first one was used for planning the position of catheters, and then the treatment planning was completed by a second imaging performed after the implantation. First, lumpectomy cavity, PTV, and organs at risk were delineated and then, the catheters were reconstructed using the $\mathrm{CT}$ information. After that, the source dwell positions were activated inside the PTV with 0 margin, and geometrical optimization was performed. Basal dose points were created in the central plane and after normalization, an isodose line was selected for dose prescription, so that the target coverage be at least $90 \%$. The f-factor used for dose prescription ranged between 0.75 and 0.90 , corresponding to isodose level of $75 \%$ and $90 \%$. At the same time, a proper dose homogeneity had to be maintained with the DNR $\leq 0.35$. At conflicting values, the target volume coverage was prioritized. In case when the target coverage was not sufficient, graphical optimization was applied to improve the dose distribution locally in the underdosed regions. The prescribed dose (PD) was $7 \times 4.3 \mathrm{~Gy}$. This 
method is called conventional or forward planning, and referred as GO + GRO. The catheters were implanted into the breast in a triangular pattern, with a separation of $1.3 \mathrm{~cm}$. The source step size was $2.5 \mathrm{~mm}$, the first/last source position in the catheters was always at least $5 \mathrm{~mm}$ from the skin surface. The resolution of calculation matrix was $1 \mathrm{~mm}$.

\section{Inverse planning}

Retrospectively, for each patient, a plan with inverse optimization (HIPO) was made using the same CT images and contours used in forward planning. The input parameters in HIPO that were used included: for PTV MinWeight $=75$, MinValue $=100$, MaxWeight $=25$, and MaxValue $=150$, and for normal tissue - MaxWeight $=5$ and MaxValue $=120$. Normal tissue, which is always mandatory for the optimization, is considered as all tissues surrounding the PTV, excluding any other OARs. For other OARs, no objectives were applied here. The MinValue and MaxValue were related to the PD as percentages. The value for DTGR parameter was selected to be 0.2. The active source positions were inside the PTV, similarly to GO + GRO.

Competing plans were assessed and compared with dose-volume parameters, such as relative volumes receiving a percentage of $\mathrm{PD}$ (e.g., $\mathrm{V}_{100}, \mathrm{~V}_{90}, \mathrm{~V}_{50}$ ) and relative doses in percentage of the PD to small absolute volumes (e.g., $\left.\mathrm{D}_{0.1 \mathrm{~cm} 3}, \mathrm{D}_{1 \mathrm{~cm} 3}\right)$. Since the skin and ribs were not delineated as whole organs, the $V_{5}$ for skin and the $V_{50}$ for ribs were given as absolute value in $\mathrm{cm}^{3}$. The dose homogeneity was described by the DNR (dose non-uniformity ratio) and the conformality with COIN (conformity index). The COIN simultaneously considers the coverage of PTV and irradiation of normal tissues outside the PTV by the PD [24]. Descriptive statistics were used to characterize the two groups of treatment plans, and the distribution of parameters was tested with Shapiro-Wilk's $\mathrm{W}$ test regarding normality. Since most of the parameters were not normally distributed, non-parametric Wilcoxon

A

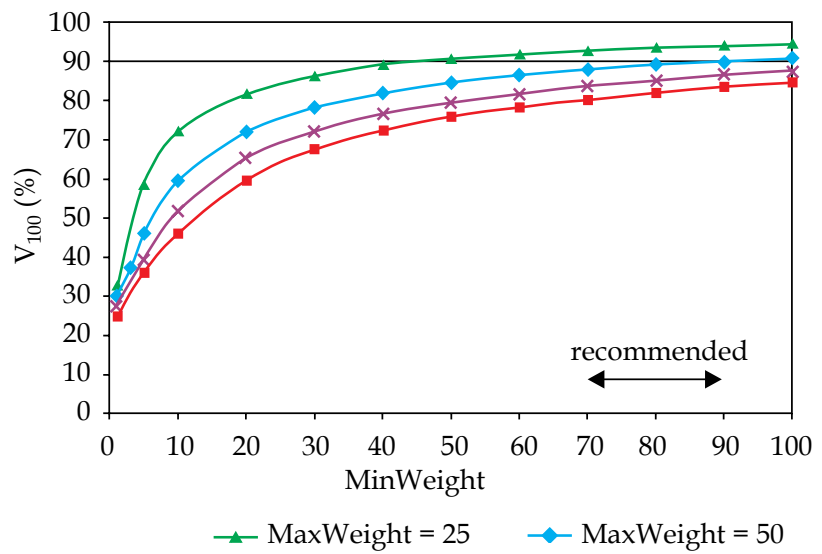

matched-pairs test was used for all comparisons with Statistica 7.0 software (StatSoft, Inc., Tulsa, OK, USA), and $p<0.05$ was considered statistically significant.

\section{Results}

A representative patient with a PTV of $34 \mathrm{~cm}^{3}$ and implanted with 12 catheters was selected to study the effect of optimization parameters in HIPO.

\section{Target coverage and dose homogeneity}

In Figure 1, the target coverage and dose homogeneity are presented in the function of minimum weight at different homogeneity parameters. As the minimum weight increased, the $\mathrm{V}_{100}$ continuously improved. The larger the MinWeight, the higher the $\mathrm{V}_{100}$ (Figure 1A). Furthermore, the maximal coverage developed when the constraint for dose homogeneity was weak (MaxWeight $=25$ ). When the homogeneity was the greatest (MaxWeight $=100$ ), the coverage was less than $80 \%$. The dependence of DNR on MinWeight was not monotonous (Figure 1B). As the MinWeight increased, the DNR decreased first and then slowly increased. The lowest DNR was around 0.20 but developed at different MinWeight depending on the MaxWeight. Based on the graphs in Figure 1A, the value for minimum weight in the range of 70-90 seemed to be an acceptable compromise between the target coverage and the dose homogeneity.

Looking at the changes of $\mathrm{V}_{100}$ and DNR in the function of dose homogeneity parameter (MaxWeight), an explicit dependence was observed. Putting emphasis on the importance of dose homogeneity both the $V_{100}$ and the DNR decreased (Figure 2). The DNR was the lowest at MinWeight $=25$ (Figure 2B), but the coverage was not acceptable at MaxWeight higher than 10. The $\mathrm{V}_{100}$ was greatly influenced by the MinWeight and the MaxWeight, which is shown in Figure 2A. Only low values of MaxWeight resulted in adequate coverage $\left(\mathrm{V}_{100} \geq 90 \%\right)$, but the DNR was high at MaxWeight below 10. A range

\section{B}

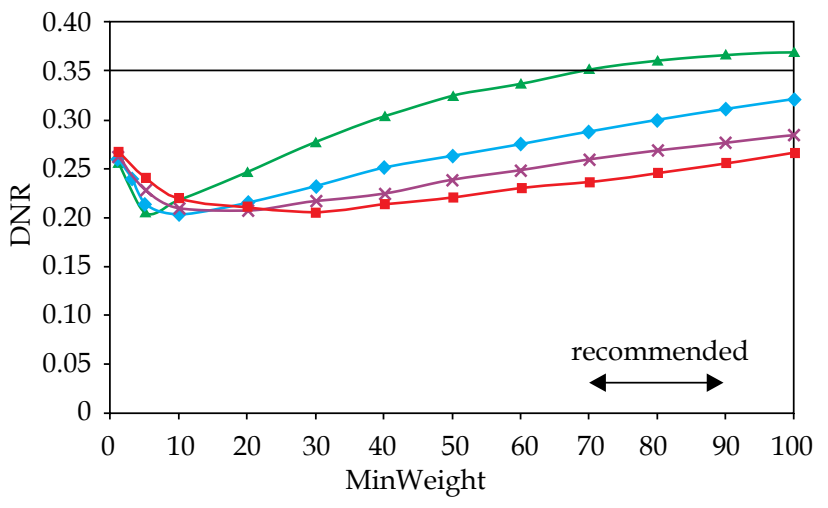

$\leftarrow$ MaxWeight $=75 \rightarrow$ MaxWeight $=100$

Fig. 1. Dependence of target coverage (A) and dose homogeneity (B) on the weight factor for minimum dose (MinWeight) at the periphery of PTV. The horizontal line shows the planning aim and the double-headed arrow indicates the range of recommended values. $\mathrm{V}_{100}$ is the percentage of PTV receiving the prescribed dose, DNR is the dose non-uniformity ratio. MaxWeight is a weight factor controlling the dose homogeneity in the PTV 
A

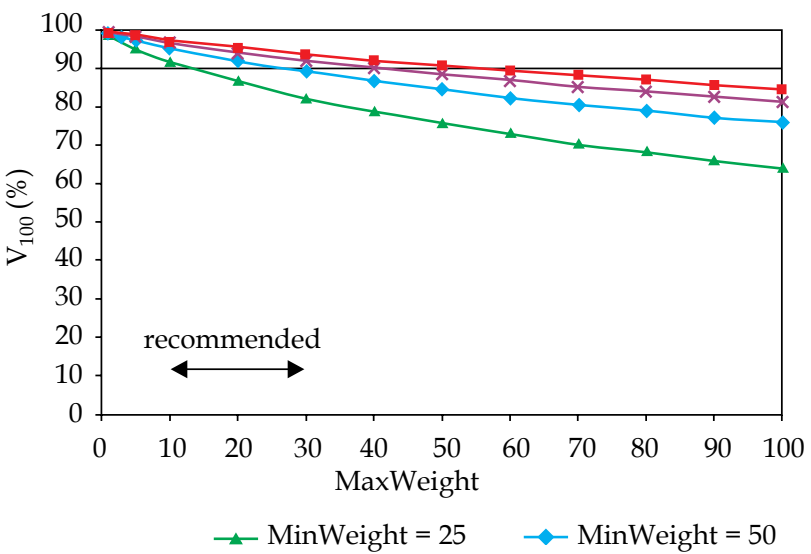

B

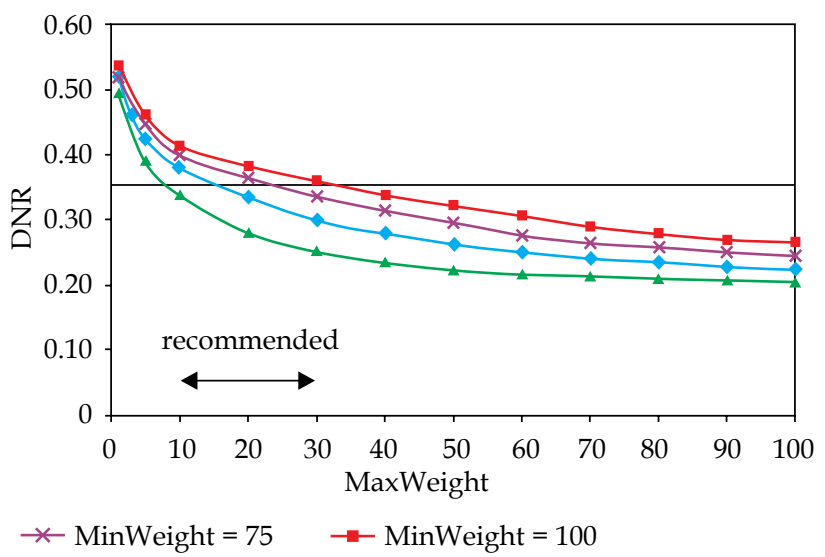

Fig. 2. Dependence of target coverage (A) and dose homogeneity (B) on the weight factor for maximum dose (MaxWeight) in the PTV. The horizontal line shows the planning aim and the double-headed arrow indicates the range of recommended values. $\mathrm{V}_{100}$ is the percentage of the PTV receiving the prescribed dose, DNR is the dose non-uniformity ratio. MinWeight is a weight factor controlling the target coverage through minimum dose at the periphery of the PTV

between 10-30 was a compromise again between the target coverage and the dose homogeneity.

\section{Normal tissue}

The normal tissue surrounded the PTV and it was always obligatory to use objectives for it. Table 1 shows how the two input parameters (MaxValue, MaxWeight) affect the target coverage characterized by the $\mathrm{V}_{100}$. The shaded area included the coverage higher than $90 \%$. At low weight factor (MaxWeight $=5$ ), the $V_{100}$ was always larger than $90 \%$ independently on the maximal dose value (MaxValue). If the allowed dose maximum was $120 \%$, the weight factor did not influence the coverage, since the $\mathrm{V}_{100}$ was between $91.7 \%$ and $92.7 \%$. The dose homogeneity also depended on MaxValue and MaxWeight of normal tissue (Table 2). In the shaded area, the DNR was less than 0.35 . If the MaxValue was relatively low $(\leq 60 \%)$ homogeneous dose distribution developed at any weight factor (DNR < 0.35). When using a low weight factor for maximum dose (MaxWeight $=5$ ), the DNR was always low (0.30-0.31) and it did not depend on the maximum dose value. Summarizing the results shown in Tables 1

Table 1. Effect of MaxValue and MaxWeight parameters of normal tissue on $\mathrm{V}_{100}(\%)$

\begin{tabular}{lccccc} 
Max & \multicolumn{5}{c}{ MaxWeight } \\
\cline { 2 - 6 } Value & 5 & 25 & 50 & 75 & 100 \\
\hline 20 & 90.6 & 82.1 & 65.2 & 46.6 & 28.3 \\
\hline 40 & 90.6 & 82.2 & 67.1 & 54.6 & 47.3 \\
\hline 60 & 90.8 & 84.8 & 77.6 & 72.0 & 68.3 \\
\hline 80 & 91.6 & 89.4 & 86.3 & 84.1 & 82.7 \\
\hline 100 & 92.1 & 91.7 & 90.8 & 89.9 & 89.4 \\
\hline 120 & 92.7 & 92.7 & 92.2 & 92.0 & 91.7
\end{tabular}

$V_{100}$ - percentage of the PTV receiving the prescribed dose and 2, the MaxValue = 120 with MaxWeight $=5$ as an objective for normal tissue seemed to be the right selection for input parameters in inverse planning.

\section{Organs at risk}

With proper normal tissue dosimetry, the dose to OARs can be kept low. If an OAR was not used directly during dose optimization, it would be a part of normal tissue and the dose objectives to normal tissue would implicitly apply to that as well. For example, if the target is superficially located and the PTV touches the skin defined as a $5 \mathrm{~mm}$ layer from body surface, the dose objective set up for normal tissue will govern the skin dosimetry. This is demonstrated in Table 3, since the coverage was nearly identical at all weight factors and change of the DNR was also very small. When the maximum dose to skin was increased from $40 \%$ to $120 \%$ at maximum weight factor (MaxWeigh $=100)$, the coverage improved slightly and the homogeneity marginally deteriorated, but the change of $\mathrm{V}_{100}$ and DNR was less than 1\% (Table 4). In this analysis, we used MaxValue $=120$ and MaxWeight $=5$ for normal tissue.

Table 2. Effect of MaxValue and MaxWeight parameters of normal tissue on DNR

\begin{tabular}{lccccc} 
Max & \multicolumn{5}{c}{ MaxWeight } \\
\cline { 2 - 6 } Value & 5 & 25 & 50 & 75 & 100 \\
\hline 20 & 0.30 & 0.33 & 0.27 & 0.26 & 0.27 \\
\hline 40 & 0.31 & 0.33 & 0.28 & 0.26 & 0.28 \\
\hline 60 & 0.30 & 0.34 & 0.33 & 0.31 & 0.31 \\
\hline 80 & 0.31 & 0.36 & 0.36 & 0.36 & 0.36 \\
\hline 100 & 0.30 & 0.36 & 0.37 & 0.37 & 0.37 \\
\hline 120 & 0.30 & 0.36 & 0.36 & 0.36 & 0.37
\end{tabular}

$D N R$ - dose non-uniformity ratio 
Table 3. Effect of weight factor of maximum dose to skin on target coverage and dose homogeneity $($ MaxValue $=120)$

\begin{tabular}{lll} 
MaxWeight & $\mathrm{V}_{100}$ & DNR \\
\hline 0.001 & 92.66 & 0.300 \\
\hline 1 & 92.66 & 0.302 \\
\hline 10 & 92.67 & 0.304 \\
\hline 20 & 92.59 & 0.300 \\
\hline 40 & 92.54 & 0.303 \\
\hline 60 & 92.54 & 0.303 \\
\hline 80 & 92.53 & 0.307 \\
\hline 100 & 92.55 & 0.308
\end{tabular}

$V_{100}$-percentage of the PTV receiving the prescribed dose, DNR-dose non-uniformity ratio

Table 4. Effect of maximum dose to skin on target coverage and dose homogeneity (MaxWeight $=100$ )

\begin{tabular}{lll} 
MaxValue & $\mathrm{V}_{100}$ & DNR \\
\hline 40 & 91.67 & 0.304 \\
\hline 60 & 92.16 & 0.304 \\
\hline 80 & 92.49 & 0.305 \\
\hline 100 & 92.55 & 0.306 \\
\hline 120 & 92.55 & 0.307
\end{tabular}

$V_{100}$ - percentage of the PTV receiving the prescribed dose, DNR-dose non-uniformity ratio

Table 5. Effect of dwell time gradient restriction (DTGR) on target coverage and dose homogeneity

\begin{tabular}{lll} 
DTGR & $V_{100}$ & DNR \\
\hline 0.0 & 93.7 & 0.341 \\
\hline 0.1 & 93.4 & 0.354 \\
\hline 0.2 & 93.3 & 0.358 \\
\hline 0.3 & 93.1 & 0.355 \\
\hline 0.4 & 93.2 & 0.354 \\
\hline 0.5 & 93.2 & 0.354 \\
\hline 0.6 & 93.2 & 0.353 \\
\hline 0.7 & 93.2 & 0.356 \\
\hline 0.8 & 93.2 & 0.355 \\
\hline 0.9 & 93.2 & 0.357 \\
\hline 1.0 & 93.2 & 0.359
\end{tabular}

$V_{100}$ - percentage of the PTV receiving the prescribed dose, DNR-dose non-uniformity ratio

\section{Dwell time gradient restriction (DTGR)}

Table 5 shows the effects of DTGR on target coverage and dose homogeneity. When no constraint was used for neighboring dwell times (DTGR $=0.0$ ), the DNR was the lowest (0.341) and the $V_{100}$ the highest (93.7). From the data, it can be observed that in the function of DTGR, the variation of both parameters was small, less than $1 \%$ for the $\mathrm{V}_{100}$ and less than $5 \%$ for the DNR.

\section{Patient dosimetry}

The mean volume of lumpectomy cavity and PTV was $10.7 \mathrm{~cm}^{3}$ (range, 3.5-37.0 $\mathrm{cm}^{3}$ ) and $56.7 \mathrm{~cm}^{3}$ (range, 26.6$\left.173.6 \mathrm{~cm}^{3}\right)$, respectively. Twenty-seven patients $(64 \%)$ had left-sided and fifteen (36\%) right-sided lesion. In seven cases (17\%), the PTV was close to and in eight cases (19\%) far from the skin. Median 13 catheters were implanted into the breast, with a range between 7 and 28 . Table 6 includes dosimetric parameters for treatment plans made by forward (GO + GRO) and inverse (HIPO) planning for forty-two patients. The basis of comparison was the same target coverage. Both, the mean $V_{100}$ and $\mathrm{D}_{90}$ were nearly identical for the two groups. But the conformality and the homogeneity was also better with the inverse planning. The mean COIN increased from 0.69 to $0.75(p<0.0001)$ and the DNR decreased from 0.39 to 0.35 $(p<0.0027)$. The volume irradiated by the PD was significantly smaller in the inverse plans $\left(63.6 \mathrm{~cm}^{3}\right.$ vs. 67.5 $\mathrm{cm}^{3}, p<0.0001$ ). The non-target breast (ipsilateral breast excluding the PTV) received significantly less dose in the HIPO plans, and the same applied for the ipsilateral lung. The mean lung dose (MLD) was $8 \%$ less in HIPO and in the other three parameters $\left(D_{0.1 \mathrm{~cm} 3}, D_{1 \mathrm{~cm} 3}, V_{5}\right)$, there was a $5 \%$ decrease as well. Although, the doses to the most exposed $0.1 \mathrm{~cm}^{3}$ and $1 \mathrm{~cm}^{3}$ of skin were less with inverse planning, the difference was not significant. But the absolute volume irradiated by low-dose $(5 \%)$ was $5.8 \mathrm{~cm}^{3}$ less with HIPO $(p<0.0001)$. Regarding the dose to ribs, the HIPO was superior with significant less doses $\left(\mathrm{D}_{0.1 \mathrm{~cm} 3}\right.$, $\left.\mathrm{D}_{1 \mathrm{~cm} 3}\right)$, and the volume irradiated by half of the PD $\left(\mathrm{V}_{50}\right)$ was also less compared to forward planning $(1.7 \%$ vs. $1.8 \%, p=0.0247)$. We noted that the reference isodose line never reached the ribs in any HIPO plan meaning that the maximum dose was always less than the PD, and in forward plans, we observed only one case out of forty-two patients where volume of $0.1 \mathrm{~cm}^{3}$ received the PD. Twenty-seven patients had left-sided cancer, and looking at the heart dosimetry, the superiority of inverse over forward planning could be seen. The mean heart dose was $8 \%$ less, and in HIPO plans, we observed not only lower doses to most exposed small volumes $\left(0.1 \mathrm{~cm}^{3}, 1 \mathrm{~cm}^{3}\right)$, but also smaller volumes irradiated by low-dose (5\%). The amount of decrease in heart parameters ranged between $8-12 \%$, with always statistical significance. The dose to contralateral breast and lung was always small, with no significant differences between the two planning techniques.

\section{Discussion}

Inverse planning has been intensively used in external beam radiotherapy at least for a decade to optimize 
Table 6. Comparison of patient dosimetry between treatment plans made by forward (GO + GRO) and inverse (HIPO) optimization techniques for 42 patients with mean \pm standard deviation

\begin{tabular}{|c|c|c|c|c|}
\hline & $\mathrm{GO}+\mathrm{GRO}$ & HIPO & Difference $^{\#}$ & $P$-value \\
\hline \multicolumn{5}{|l|}{ PTV } \\
\hline $\mathrm{V}_{100}(\%)$ & $91.2 \pm 2.43$ & $91.4 \pm 2.48$ & 0.2 & 0.9111 \\
\hline $\mathrm{D}_{90}(\%)$ & $101.6 \pm 4.89$ & $102.0 \pm 4.21$ & 0.4 & 0.2914 \\
\hline COIN & $0.69 \pm 0.06$ & $0.75 \pm 0.07$ & 0.06 & $<0.0001$ \\
\hline \multicolumn{5}{|l|}{ Implant } \\
\hline DNR & $0.39 \pm 0.07$ & $0.35 \pm 0.05$ & -0.04 & 0.0027 \\
\hline \multicolumn{5}{|c|}{ Non-target breast } \\
\hline $\mathrm{V}_{100}(\%)$ & $2.2 \pm 1.77$ & $1.5 \pm 0.94$ & -0.7 & $<0.0001$ \\
\hline $\mathrm{V}_{50}(\%)$ & $13.0 \pm 8.46$ & $12.0 \pm 7.65$ & -1.0 & $<0.0001$ \\
\hline$V_{25}(\%)$ & $32.0 \pm 15.22$ & $30.5 \pm 14.56$ & -1.5 & $<0.0001$ \\
\hline \multicolumn{5}{|c|}{ Ipsilateral lung } \\
\hline MLD (\%) & $5.3 \pm 2.18$ & $4.9 \pm 1.63$ & -0.4 & $<0.0001$ \\
\hline$\underline{\mathrm{D}_{0.1 \mathrm{~cm}^{3}}(\%)}$ & $42.9 \pm 13.99$ & $40.6 \pm 12.98$ & -2.3 & $<0.0001$ \\
\hline $\mathrm{D}_{1 \mathrm{~cm} \mathrm{~m}^{3}}(\%)$ & $37.7 \pm 12.61$ & $35.7 \pm 11.78$ & -2.0 & $<0.0001$ \\
\hline$V_{5}(\%)$ & $31.9 \pm 10.29$ & $30.3 \pm 10.11$ & -1.6 & $<0.0001$ \\
\hline \multicolumn{5}{|l|}{ Skin } \\
\hline$\underline{\mathrm{D}_{0.1 \mathrm{~cm}^{3}}(\%)}$ & $76.1 \pm 30.03$ & $73.8 \pm 30.39$ & -2.3 & 0.1027 \\
\hline $\mathrm{D}_{1 \mathrm{~cm}^{3}}(\%)$ & $59.1 \pm 19.82$ & $57.5 \pm 19.65$ & -1.6 & 0.1633 \\
\hline $\mathrm{V}_{5}\left(\mathrm{~cm}^{3}\right)$ & $173.5 \pm 47.65$ & $167.7 \pm 45.75$ & -5.8 & $<0.0001$ \\
\hline \multicolumn{5}{|l|}{ Ribs } \\
\hline$\underline{\mathrm{D}_{0.1 \mathrm{~cm}^{3}}(\%)}$ & $56.6 \pm 19.21$ & $52.1 \pm 18.82$ & -4.5 & $<0.0001$ \\
\hline$\underline{\mathrm{D}}_{1 \mathrm{~cm} \mathrm{~m}^{3}}(\%)$ & $44.8 \pm 16.73$ & $41.7 \pm 16.76$ & -3.1 & $<0.0001$ \\
\hline $\mathrm{V}_{50}\left(\mathrm{~cm}^{3}\right)$ & $1.8 \pm 2.87$ & $1.7 \pm 3.15$ & -0.1 & 0.0247 \\
\hline \multicolumn{5}{|l|}{ Heart* } \\
\hline MHD (\%) & $3.9 \pm 1.79$ & $3.6 \pm 1.75$ & -0.3 & $<0.0001$ \\
\hline$\underline{\mathrm{D}_{0.1 \mathrm{~cm}^{3}}(\%)}$ & $22.0 \pm 11.86$ & $19.4 \pm 11.14$ & -2.6 & 0.0016 \\
\hline $\mathrm{D}_{1 \mathrm{~cm} \mathrm{~m}^{3}}(\%)$ & $18.2 \pm 10.06$ & $16.6 \pm 9.77$ & -1.6 & 0.0002 \\
\hline$V_{5}(\%)$ & $25.8 \pm 17.89$ & $23.7 \pm 17.95$ & -2.1 & 0.0008 \\
\hline \multicolumn{5}{|c|}{ Contralateral breast } \\
\hline$\underline{\mathrm{D}_{0.1 \mathrm{~cm}^{3}}(\%)}$ & $4.3 \pm 2.9$ & $3.8 \pm 2.55$ & -0.5 & 0.5165 \\
\hline $\mathrm{D}_{1 \mathrm{~cm} \mathrm{~m}^{3}}(\%)$ & $2.9 \pm 1.79$ & $2.6 \pm 1.68$ & -0.3 & 0.1976 \\
\hline \multicolumn{5}{|c|}{ Contralateral lung } \\
\hline$\underline{\mathrm{D}_{0.1 \mathrm{~cm}^{3}}(\%)}$ & $4.9 \pm 2.81$ & $5.2 \pm 2.72$ & 0.3 & 0.3131 \\
\hline $\mathrm{D}_{1 \mathrm{~cm} \mathrm{~m}^{3}}(\%)$ & $3.5 \pm 1.82$ & $3.5 \pm 1.92$ & 0.0 & 0.9739 \\
\hline
\end{tabular}

GO - geometrical optimization, GRO - graphical optimization, HIPO - hybrid inverse planning optimization; *only for left-sided lesions (27 patients), \#HIPO $-(G O+G R O)$

the dose distribution for state-of-the-art dose delivery techniques, such as intensity modulated radiation therapy and volume modulated arc therapy. However, in brachytherapy, it is still not routinely used for various treatment sites, except for the prostate. In clinical practice in both LDR and HDR prostate BT, optimal positions of radioactive sources are determined with inverse planning for no less than two decades, considering surface and volumetric dose constraints in addition to dose limits to urethra and rectum $[6,21,25,26,27,28,29,30,31]$. The role of inverse planning in gynecological BT is also studied in the literature. Trnková et al. [7] analyzed the feasibility of inverse planning algorithm (HIPO) for image-guided cer- vical cancer brachytherapy. Ten patients treated with intracavitary and another ten with combined intracavitary/ interstitial techniques were planned with manual and inverse optimization. They found that HIPO was feasible for improving the therapeutic ratio and limiting the substantial high-dose regions around the needles; therefore, it was introduced into their clinical practice. The same group made a comparison between manual and inverse plans in HDR intracavitary/interstitial cervical cancer brachytherapy using data of 20 patients [8]. They found that HIPO produced clinically acceptable treatment plans and enabled the reduction of high-dose regions in the immediate vicinity of the target volume. Compared to 
IPSA, HIPO considered the current clinical experience gained from manual treatment planning. Other authors also found inverse planning feasible for cervical cancer BT $[9,10,11,12,31]$. Inverse planning can offer good sparing of critical structures without compromising the target coverage [9]. Moreover, IPSA significantly reduces overall treatment planning time with improved reduction of doses to the OARs, as compared with a volume-based optimization treatment planning method [12]. Matias et al. [11] provided a fine class solution of HIPO, demonstrating comparable results in relation to graphical optimization regarding both dosimetric and radiobiological endpoints. Kannan et al. [10] found that IPSA significantly improved the target volume coverage compared with manual planning. Inverse planning in vaginal BT performed with multichannel applicator was also studied, and its dosimetric advantages were demonstrated using planning data in two studies with twelve patients in both investigations [14,15]. For other sites, information about inverse planning in BT is sparsely available. In a case study with two patients, Jameson et al. [16] presented the usefulness of IPSA for head and neck BT, and Choi et al. [17] compared two inverse algorithms (IPSA, HIPO) in interstitial tongue HDR-BT of 20 patients. Regarding breast cancer, two inverse planning algorithms (IPSA, HIPO) were compared for 20 patients treated with BT boost using rigid needles [32]. With IPSA algorithm, the dose to OARs was less, but the target coverage and conformality was better with HIPO. In a recent paper, simulated annealing optimization was demonstrated for permanent breast implant treatment planning [33]. Treatment plans produced manually and with inverse planning of a 10-patient cohort were dosimetrically compared. Although in target coverage and dose to skin, no difference was found, a statistical difference was observed in reduction of volume irradiated by the 1.5 times the PD and in increase of conformity index in favor of inverse planning.

To the best of our knowledge, in interstitial HDR breast BT, detailed investigation of the input parameters of inverse optimization algorithms on dosimetry has not been performed. In our study, using HIPO, we firstly analyzed the effect of weight factors on target dose parameters accountable for target coverage (MinWeight), and dose homogeneity (MaxWeight) on quality of dose distributions in a breast implant. We clearly showed that these two factors played against each other. In order to find an acceptable compromise between the coverage and the homogeneity, we changed those weight factors stepby-step and subsequently determined a recommended range of their values. Dosimetric constraints for normal tissues surrounding the PTV had an influence on both the coverage and the homogeneity. To investigate this phenomenon, we changed the maximum dose (MaxValue between $20-120 \%$ ) and its weight (MaxWeight between 5-100) to normal tissue and calculated the $V_{100}$ and the DNR. According to data in Table 1, the weight factor has a role only when the prescribed maximum dose in normal tissue is low $(\leq 100 \%)$. If the MaxValue is 120 , the $V_{100}$ is always larger than $90 \%$, independently on the weight factor. At low maximum doses (MaxValue $\leq 60 \%$ ), the dose distribution is always homogeneous with low DNR, and the weight factor does not have a big effect (Table 2). But, at low weight factor (MaxWeight $=5$ ), the DNR is practically the same (0.30-0.31) at any maximum dose value.

The skin is considered as one of the most important OARs in breast BT. Similarly, to normal tissue, a maximum dose and its weight factor are the input parameters for skin dosimetry in inverse planning. Logically, with these two parameters, the dose to skin can be simply controlled. But, at the same time, the target coverage and the dose homogeneity are changing as well. If the skin is far from the PTV, there is no reason to use dose constraints, since it always receives relatively low dose. However, when the PTV is in close proximity to the skin, dose objectives in order to decrease the maximum dose can be applied. It is evident that the smaller the distance between the skin and PTV, the higher the maximum dose to skin. We investigated the role of input parameters of optimization for skin when the PTV is in contact with skin. Tables 3 and 4 demonstrate how the $V_{100}$ and DNR are affected by the values of MaxWeight and MaxValue. Summarizing the results obtained with optimization of dose to skin, we can state that, basically, there is no effect of dose objectives for skin on the target coverage and the dose homogeneity. The explanation can be that the optimization objectives for normal tissue are strong enough to control the dose distribution outside the PTV. In fact, if it is not outlined separately, the skin is a part of normal tissue and if similar dose constraints are used as for the normal tissue, their effects will be minimal. Considering that the positions of the ribs related to the PTV can be very similar to the skin (far from or close to it), the conclusions drawn for skin dosimetry are valid for the ribs as well. We recommend using optimization parameters for any OAR only in a special case, when the maximum dose has to be kept below a certain level. Nevertheless, it must not be forgotten that dose to any OAR cannot be reduced unless the dose delivered to the PTV is decreased.

The classical dosimetry systems are based on LDR sources. With the stepping source technique, those systems can be simulated using uniform source dwell times $[2,3,34]$. However, after any optimization technique, the dwell times will be non-uniform, occasionally with large fluctuation. The dwell time gradient restriction in HDR stepping source dosimetry was introduced to avoid large dwell time differences in adjacent source dwell positions [35]. The role of controlling neighboring dwell times in quality of treatment plan is controversial. In a recent study, a negative correlation was found between modulation restriction, and both HR-CTV $D_{90}$ and $V_{100}$ in cervix BT as well as an increasing restriction, negatively affected the conformity index [36]. According to the results of Mavroidis et al. [37], dwell time regularization technique in HIPO introduced a minor improvement in the effectiveness of the optimized prostate HDR dose distributions. Balvert et al. [38] also investigated the role of dwell time modulation restrictions for HDR prostate BT and found that the use of restriction did not necessarily improve the treatment plan quality. In another study, an increase of dwell time deviation constraint yielded a few percent decrease in target coverage and a slight increase in $\mathrm{V}_{150}$ in HDR prostate implants using IPSA [39]. The lat- 
ter is in agreement with our results shown in Table 5. The most homogeneous dose distribution develops when no restriction is used for the gradient, and the DNR is continuously increasing with stronger restriction. However, the difference between the extreme DNR values is only $5 \%$, while the value of $\mathrm{V}_{100}$ practically does not change.

Although our detailed investigation about the role of input parameters on plan quality in inverse planning was performed only in one selected case, the results were validated in a relatively large patient cohort of forty-two cases. Our data showed that inverse planning performs better than forward planning regarding dosimetric parameters of target and OARs. In interstitial breast implants, a large number of dwell positions are in the target volume, which probably contributes to the efficacy of optimization algorithm. In other treatment sites such as cervix or head and neck, much less dwell positions are used, and the advantages of inverse planning may not be so evident. Though quantitative data about planning time was not given here, we noted that inverse planning requires considerably less amount of time than forward planning. This is another point for implementing inverse planning in clinical practice.

\section{Conclusions}

Prerequisite for effective use of inverse planning algorithm in interstitial breast BT is the determination of proper input parameters for optimization. Having found those presets, the optimization procedure is fast and practically always resulting in more superior dose distribution, when compared with forward planning. This statement was verified by comparing the data of patients' dosimetry obtained by the two optimization methods. Our results clearly show that inverse planning is superior to forward planning, since at the same target coverage, all dosimetric parameters for any OAR, except only for contralateral lung, were more favorable with inverse planning, and in most cases, the differences were statistical significant. Furthermore, more homogeneous and more conformal dose distributions developed after inverse planning. Based on our results, the introduction of inverse planning into routine clinical practice is recommended in interstitial HDR breast brachytherapy.

\section{Acknowledgements}

Financial support from the 2019 Thematic Excellence Program (TUDFO/51757/2019-ITM) is greatly acknowledged.

\section{Disclosure}

The authors report no conflict of interest.

\section{References}

1. Major T, Polgár C, Lövey K et al. Dosimetric characteristics of accelerated partial breast irradiation with CT image-based multicatheter interstitial brachytherapy: A single institution's experience. Brachytherapy 2011; 10: 421-426.

2. Wiercinska J, Wronczewska A, Kabacinska R et al. Transition from Paris dosimetry system to 3D image-guided planning in interstitial breast brachytherapy. J Contemp Brachytherapy 2015; 7: 479-484

3. Major T, Polgár C. Treatment planning for multicatheter interstitial brachytherapy of breast cancer - from Paris system to anatomy-based inverse planning. I Contemp Brachytherapy 2017; 9: 89-98.

4. Demianovich A, Sanin D, Martynova V et al. Experience in multicatheter interstitial high-dose-rate breast brachytherapy: dose-volume histogram analysis of the first results. J Contemp Brachytherapy 2019; 11: 349-355.

5. Karabis A, Giannouli S, Baltas D. HIPO: a hybrid inverse treatment planning optimization algorithm in HDR brachytherapy. Radiother Oncol 2005; 76: S29.

6. Lessard E, Pouliot J. Inverse planning anatomy-based dose optimization for HDR-brachytherapy of the prostate using fast simulated annealing algorithm and dedicated objective function. Med Phys 2001; 28: 773-779.

7. Trnková P, Pötter R, Baltas D et al. New inverse planning technology for image-guided cervical cancer brachytherapy: Description and evaluation within a clinical frame. Radiother Oncol 2009; 93: 331-340.

8. Trnková P, Baltas D, Karabis A et al. A detailed dosimetric comparison between manual and inverse plans in HDR intracavitary/ interstitial cervical cancer brachytherapy. J Contemp Brachytherapy 2010; 2: 163-170.

9. Jamema SV, Kirisits C, Mahantshetty U et al. Comparison of DVH parameters and loading patterns of standard loading, manual and inverse optimization for intracavitary brachytherapy on a subset of tandem/ovoid cases. Radiother Oncol 2010; 97: 501-506.

10. Kannan RA, Gururajachar JM, Ponni A et al. Comparison of manual and inverse optimisation techniques in high dose rate intracavitary brachytherapy of cervical cancer: A dosimetric study. Rep Pract Oncol Radiother 2015; 20: 365-369.

11. Matias Ldos S, Palmqvist T, Wolke J et al. Dosimetric and radiobiological evaluation of hybrid inverse planning and optimization for cervical cancer brachytherapy. Anticancer Res 2015; 35: 6091-6096.

12. Pelagade S, Maddirala HR, Misra R et al. Dosimetric comparison of volume-based and inverseplanning simulated annealing-based dose optimizations for high-dose-rate brachytherapy. Med Dosim 2015; 40: 235-239.

13. Tang B, Liu X, Wang $X$ et al. Dosimetric comparison of graphical optimization and inverse planning simulated annealing for brachytherapy of cervical cancer. J Contemp Brachytherapy 2019; 11: 379-383.

14. Bahadur YA, Constantinescu C, Hassouna AH et al. Single versus multichannel applicator in high-dose-rate vaginal brachytherapy optimized by inverse treatment planning. J Contemp Brachytherapy 2014; 6: 362-370.

15. Carrara M, Cusumano D, Giandini T et al. Comparison of different treatment planning optimization methods for vaginal HDR brachytherapy with multichannel applicators: A reduction of the high doses to the vaginal mucosa is possible. Phys Med 2017; 44: 58-65.

16. Jameson MG, Ohanessian L, Batumalai V et al. Comparison of Oncentra ${ }^{\circledR}$ Brachy IPSA and graphical optimisation techniques: a case study of HDR brachytherapy head and neck and prostate plans. J Med Radiat Sci 2015; 62: 168-174.

17. Choi CH, Park SY, Park JM et al. Comparison of the IPSA and HIPO algorithms for interstitial tongue high-dose-rate brachytherapy. PLoS One 2018; 13: e0205229.

18. Polgár Cs, Major T, Fodor J et al. Accelerated partial-breast irradiation using high-dose-rate interstitial brachytherapy: 12-year update of a prospective clinical study. Radiother Oncol 2010; 94: 274-279.

19. Polgár C, Fodor J, Major T et al. Breast conserving therapy with partial or whole breast irradiation: Ten-year results of 
the Budapest randomized trial. Radiother Oncol 2013; 108 : 197-202.

20. Strnad V, Ott OJ, Hildebrandt G et al. 5-year results of accelerated partial breast irradiation using sole interstitial multicatheter brachytherapy versus whole-breast irradiation with boost after breast-conserving surgery for low-risk invasive and in-situ carcinoma of the female breast: a randomized, phase 3, non-inferiority trial. Lancet 2016; 387: 229-238.

21. Karabis A, Belotti P, Baltas D. Optimization of catheter position and dwell time in prostate HDR brachytherapy using HIPO and linear programming. IFMBE Proceedings 2009; 25/1: 612-615.

22. Polgár $C$, van Limbergen E, Pötter R et al. Patient selection for accelerated partial breast irradiation (APBI) after breast conserving surgery: Recommendations of the Groupe Européen de Curiethérapie-European Society for Therapeutic Radiology and Oncology (GEC-ESTRO) Breast Cancer Working Group based on clinical evidence (2009). Radiother Oncol 2010; 94: 264-273.

23. Major T, Gutiérrez C, Guix B et al. On behalf of Breast Cancer Working Group of GEC-ESTRO. Recommendations from GEC ESTRO Breast Cancer Working Group (II): Target definition and target delineation for accelerated or boost Partial Breast Irradiation using multicatheter interstitial brachytherapy after breast conserving open cavity surgery. Radiother Oncol 2016; 118: 199-204.

24. Baltas D, Kolotas C, Geramani K et al. A conformal index (COIN) to evaluate implant quality and dose specification in brachytherapy. Int J Radiat Oncol Biol Phys 1998; 40: 515-524.

25. Messing EM, Zhang JBY, Rubens DJ et al. Intraoperative optimized inverse planning for prostate brachytherapy: early experience. Int J Radiat Oncol Biol Phys 1999; 44: 801-808.

26. Jacob D, Raben A, Sarkar A et al. Anatomy-based inverse planning simulated annealing optimization in high-dose-rate prostate brachytherapy: significant dosimetric advantage over other optimization techniques. Int J Radiat Oncol Biol Phys 2008; 72: 820-827.

27. Pokharel S, Rana S, Blikenstaff J et al. Evaluation of hybrid inverse planning and optimization (HIPO) algorithm for optimization in real-time, high-dose-rate (HDR) brachytherapy for prostate. J Appl Clin Med Phys 2013; 14: 4198.

28. Siebert FA, Wolf $\mathrm{S}$, Bertermann $\mathrm{H}$ et al. Introduction of inverse dose optimization for ultrasound-based high-dose-rate boost brachytherapy: How we do it in Kiel. Brachytherapy 2014; 13: 250-256.

29. Deis TM, Gorissen BL. High-dose-rate prostate brachytherapy inverse planning on dose-volume criteria by simulated annealing. Phys Med Biol 2016; 61: 1155-1170.

30. Poulin E, Varfalvy N, Aubin S et al. Comparison of dose and catheter optimization algorithms in prostate high-dose-rate brachytherapy. Brachytherapy 2016; 15: 102-111.

31. Fröhlich G, Geszti Gy, Vizkeleti J et al. Dosimetric comparison of inverse optimisation methods versus forward optimisation in HDR brachytherapy of breast, cervical and prostate cancer. Strahlenther Onkol 2019; 195: 991-1000.

32. Dymnicka M, Kociemba A, Krupka J et al. Comparison of two inverse planing algorithms HIPO and IPSA in terms of their suitability for breast brachytherapy planning. Phys Med 2018; 52: 154. P185.

33. Roumeliotis M, Yates B, Watt E et al. Demonstration of simulated annealing optimization for permanent breast seed implant treatment planning. Brachytherapy 2018; 17: 615-620.

34. Van der Laarse R. The stepping source dosimetry system as an extension of the Paris system. In: Brachytherapy from radium to optimization. Mould RF, Battermann JJ, Martinez AA, Speiser BL (eds.). Nucletron BV, Veenendaal 1994; pp. 319-330.
35. Baltas D, Katsilieri Z, Kefala V et al. Influence of modulation restriction in inverse optimization with HIPO of prostate implants on plan quality: analysis using dosimetric and radiobiological indices. IFMBE Proc 2009; 25: 283-286.

36. Mosleh-Shirazi MA, Shahcheraghi-Motlagh E, Gholami $\mathrm{MH}$ et al. Influence of dwell time homogeneity error weight parameter on treatment plan quality in inverse optimized high-dose-rate cervix brachytherapy using SagiPlan. J Contemp Brachytherapy 2019; 11: 256-266.

37. Mavroidis P, Katsileri Z, Kefala V et al. Radiobiological evaluation of the influence of dwell time modulation restriction in HIPO optimized HDR prostate brachytherapy implants. J Contemp Brachytherapy 2010; 2: 117-128.

38. Balvert M, Gorissen BL, den Hertog D et al. Dwell time modulation restrictions do not necessarily improve treatment plan quality for prostate HDR brachytherapy. Phys Med Biol 2015; 60: 537-548.

39. Smith RL, Panettieri V, Lancaster $C$ et al. The influence of the dwell time deviation constraint (DTDC) parameter on dosimetry with IPSA optimisation for HDR prostate brachytherapy. Australas Phys Eng Sci Med 2015; 38: 55-61. 